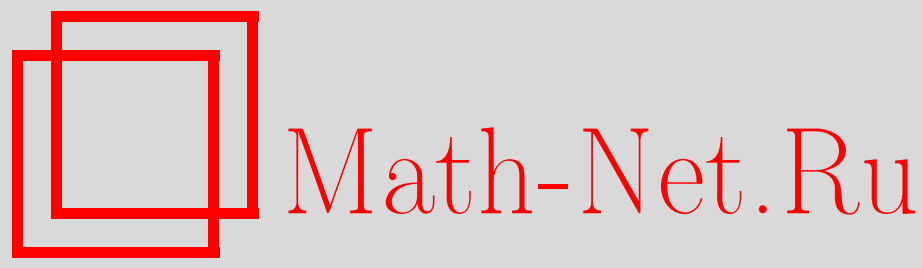

Г. П. Пронько, Проблема Кеплера в пространстве постоянной кривизны, ТМФ, 2008, том 155, номер 2, 317-326

DOI: https://doi.org/10.4213/tmf6214

Использование Общероссийского математического портала Math-Net.Ru подразумевает, что вы прочитали и согласны с пользовательским соглашением http: //www.mathnet.ru/rus/agreement

Параметры загрузки:

IP: 3.85 .183 .62

26 апреля 2023 г., 15:26:11

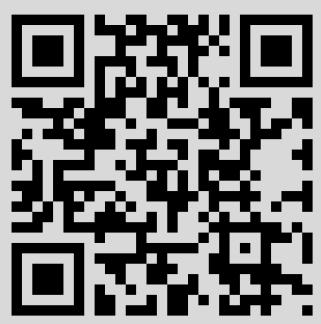




\title{
ФИЗИКА
}

Том 155, № 2

май, 2008

\section{ПРОБЛЕМА КЕПЛЕРА В ПРОСТРАНСТВЕ ПОСТОЯННОЙ КРИВИЗНЫ}

\begin{abstract}
Чисто алгебраическим путем получен спектр атома водорода в пространстве постоянной кривизны.
\end{abstract}

Ключевые слова: динамические системы, пространство постоянной кривизны, проблема Кеплера.

\section{1. ВВЕДЕНИЕ}

Теория различных механических систем в пространстве с постоянной кривизной имеет длинную историю. Особенно интересна теория атома водорода в таком пространстве, которая была впервые рассмотрена Шредингером [1] в связи с возможными космологическими применениями. Список литературы, в которой обсуждаются эти вопросы, приведен в [2]. В данной статье рассматривается динамическая симметрия атома водорода в пространстве постоянной кривизны в классическом и квантовом случаях и, как и в случае плоского пространства [3], [4], выводится формула для спектра этой системы чисто алгебраическим путем. Как оказалось, несмотря на схожесть двух систем (в плоском и искривленном пространствах), построение генераторов динамической симметрии в последнем случае весьма нетривиально.

Трехмерные пространства постоянной кривизны - это сфера $S^{3}$, гиперболоиды $H^{3}, H^{\prime 3}$ и конус $V^{3}$. Для определенности мы рассмотрим случай положительной кривизны, т.е. сферу $S^{3}$. Координаты $S^{3}$ будем обозначать $X_{\alpha}, \alpha=1,2,3,4$, и $X_{\alpha}^{2}=1$. Простейшее отображение $\mathbb{R}^{3}\left(\mathbb{R}^{3} /\{\infty\}\right)$ на $S^{3}$ дается стереографической проекцией

$$
X_{\alpha}=\left(\frac{2 \lambda \mathbf{x}}{\mathbf{x}^{2}+\lambda^{2}}, \frac{\mathbf{x}^{2}-\lambda^{2}}{\mathbf{x}^{2}+\lambda^{2}}\right) .
$$

* Институт физики высоких энергий, г. Протвино, Московская обл., Россия; Institute of Nuclear Physics, National Research Center "Demokritos", Athens, Greece.

E-mail: george.pronko@ihep.ru 
Единственный возможный кинетический член, инвариантный относительно группы движений $S O(4)$ сферы $S^{3}$, получается, если мы возьмем лагранжиан, пропорциональный квадрату тензора углового момента $M_{\alpha \beta}=X_{\alpha} \dot{X}_{\beta}-\dot{X}_{\alpha} X_{\beta}$ :

$$
L=\frac{m}{2} \frac{1}{4 \lambda^{2}} M_{\alpha \beta}^{2}=\frac{m}{2} \frac{\dot{\mathbf{x}}^{2}}{\left(\mathbf{x}^{2}+\lambda^{2}\right)^{2}} .
$$

Кинетический член в гамильтониане, соответствующем лагранжиану (2), имеет следующий вид:

$$
H=\frac{1}{2 m} \mathbf{p}^{2}\left(\mathbf{x}^{2}+\lambda^{2}\right)^{2} .
$$

Этот гамильтониан имеет шесть интегралов движения, которые являются генераторами группы движений $S O(4)$ :

$$
L_{i}=\epsilon_{i j k} x_{j} p_{k}, \quad K_{i}=\frac{1}{2 \lambda}\left(p_{i}\left(\mathbf{x}^{2}-\lambda^{2}\right)-2 x_{i} \mathbf{p x}\right) .
$$

Канонические скобки Пуассона для $p_{i}, x_{i}$ индуцируют алгебру $S O(4)$ генераторов $L_{i}, K_{i}$ :

$$
\left\{L_{i}, L_{j}\right\}=-\epsilon_{i j k} L_{k}, \quad\left\{K_{i}, K_{j}\right\}=-\epsilon_{i j k} L_{k}, \quad\left\{L_{i}, K_{j}\right\}=-\epsilon_{i j k} K_{k} .
$$

Гамильтониан $H$ пропорционален одному из операторов Казимира:

$$
H=\frac{1}{2 m} 4 \lambda^{2}\left(\mathbf{L}^{2}+\mathbf{K}^{2}\right),
$$

в то время как второй оператор Казимира обращается в нуль: $\mathbf{L K}=0$. Теперь мы добавим к гамильтониану $H$ потенциал Кеплера [1], [2]:

$$
H=\frac{1}{2 m} \mathbf{p}^{2}\left(\mathbf{x}^{2}+\lambda^{2}\right)^{2}+\frac{2 \lambda \alpha}{m} \frac{\mathbf{x}^{2}-\lambda^{2}}{|\mathbf{x}|} .
$$

Как и в плоском случае, гамильтониан (3) обладает шестью интегралами движения (разумеется, они не все независимы) - это угловой момент и вектор Лапласа-РунгеЛенца А, который имеет вид

$$
\mathbf{A}=\mathbf{K} \times \mathbf{L}+\alpha \frac{\mathbf{x}}{|\mathbf{x}|}
$$

Очевидно, что векторы $\mathbf{A}$ и $\mathbf{L}$ удовлетворяют соотношению $\mathbf{A L}=0$, а их алгебра скобок Пуассона имеет следующий вид:

$$
\left\{A_{i}, A_{j}\right\}=\epsilon_{i j k} L_{k}\left(\frac{m}{2 \lambda^{2}} H-2 \mathbf{L}^{2}\right), \quad\left\{L_{i}, A_{j}\right\}=-\epsilon_{i j k} A_{k} .
$$

Квадрат вектора $\mathbf{A}$ выражается через $\mathbf{L}^{2}$ и гамильтониан:

$$
\mathbf{A}^{2}=\alpha^{2}-\mathbf{L}^{4}+\frac{m}{2 \lambda^{2}} \mathbf{L}^{2} H
$$

Читатель, знакомый со свойствами вектора Лапласа-Рунге-Ленца в плоском случае [3], сразу увидит отличие как в алгебре, так и в связи $\mathbf{A}^{2}$ с $H$ и $\mathbf{L}^{2}$. Эти отличия 
не позволяют простым образом построить вектор, который бы образовывал вместе с $\mathbf{L}$ алгебру $S O(4)$ - алгебру динамической симметрии, “ответственной” за дополнительное вырождение в квантовой механике. Однако такое построение возможно, и, как мы увидим ниже, классическая конструкция дает направление поиска и в квантовом случае. Чтобы упростить формулы, введем масштабно-преобразованный гамильтониан $h$ :

$$
h=\frac{m}{2 \lambda^{2}} H .
$$

Из уравнения (4) получаем

$$
\mathbf{A}^{2}=-\left[\mathbf{L}^{2}-\left(\frac{h}{2}+\sqrt{\frac{h^{2}}{4}+\alpha^{2}}\right)\right]\left[\mathbf{L}^{2}-\left(\frac{h}{2}-\sqrt{\frac{h^{2}}{4}+\alpha^{2}}\right)\right]
$$

Поскольку $\mathbf{A}^{2}>0, \mathbf{L}^{2}$ удовлетворяет следующему неравенству:

$$
0 \leqslant \mathbf{L}^{2} \leqslant\left(\frac{h}{2}+\sqrt{\frac{h^{2}}{4}+\alpha^{2}}\right)
$$

Найдем теперь скобки Пуассона $\mathbf{A} f\left(\mathbf{L}^{2}\right)$ (функция $f\left(\mathbf{L}^{2}\right)$ может зависеть также и от $h)$ :

$$
\left\{A_{i} f\left(\mathbf{L}^{2}\right), A_{j} f\left(\mathbf{L}^{2}\right)\right\}=\epsilon_{i j k} L_{k} \frac{\partial\left(\mathbf{A}^{2} f^{2}\left(\mathbf{L}^{2}\right)\right)}{\partial \mathbf{L}^{2}} .
$$

Для того чтобы (6) стало частью алгебры Ли $S O(4)$, должно выполняться следующее условие:

$$
\mathbf{A}^{2} f^{2}\left(\mathbf{L}^{2}\right)=a-\mathbf{L}^{2}
$$

Очевидно, что если мы возьмем $a=\left(h / 2+\sqrt{h^{2} / 4+\alpha^{2}}\right)$, то правая часть (7) будет положительна в любой точке фазового пространства в силу неравенства (5). Более того, функция $f\left(\mathbf{L}^{2}\right)$ для такого а существенно упростится:

$$
f\left(\mathbf{L}^{2}\right)=\sqrt{\frac{a-\mathbf{L}^{2}}{\mathbf{A}^{2}}}=\left[\mathbf{L}^{2}-\left(\frac{h}{2}-\sqrt{\frac{h^{2}}{4}+\alpha^{2}}\right)\right]^{-1 / 2} .
$$

Таким образом, вектор

$$
\mathbf{R}=\mathbf{A}\left[\mathbf{L}^{2}-\left(\frac{h}{2}-\sqrt{\frac{h^{2}}{4}+\alpha^{2}}\right)\right]^{-1 / 2}
$$

вместе с угловым моментом образуют нужную алгебру $S O(4)$ :

$$
\left\{L_{i}, L_{j}\right\}=-\epsilon_{i j k} L_{k}, \quad\left\{R_{i}, R_{j}\right\}=-\epsilon_{i j k} L_{k}, \quad\left\{L_{i}, R_{j}\right\}=-\epsilon_{i j k} R_{k} .
$$

Далее нетрудно получить следующее выражение для нетривиального оператора Казимира алгебры $S O(4)$ :

$$
\mathbf{R}^{2}+\mathbf{L}^{2}=\left(\frac{h}{2}+\sqrt{\frac{h^{2}}{4}+\alpha^{2}}\right)
$$


второй оператор Казимира RL исчезает. Из (8) получаем

$$
h=\mathbf{R}^{2}+\mathbf{L}^{2}-\frac{\alpha^{2}}{\mathbf{R}^{2}+\mathbf{L}^{2}} .
$$

Это равенство, которое мы получили в классической механике, чрезвычайно важно и в квантовом случае. Очевидным препятствием для его квантового обобщения является некоммутативность операторов $\mathbf{A}$ и $\mathbf{L}^{2}$. Именно этой проблемой мы и займемся ниже.

\section{2. КВАНТОВАЯ ТЕОРИЯ}

Гильбертовым пространством проблемы Кеплера в пространстве постоянной положительной кривизны является пространство $L^{2}$ на $S^{3}$ со скалярным произведением

$$
\langle\phi \mid \psi\rangle=\int d^{4} X \bar{\phi}(X) \psi(X) \delta\left(X^{2}-1\right),
$$

где $X$ - это координаты четырехмерного пространства $X_{\alpha}, \alpha=1,2,3,4$. Используя отображение $\mathbb{R}^{3}$ на $S^{3}$, заданное соотношениями (1), мы можем переписать скалярное произведение (9) с точностью до несущественного сомножителя как интеграл по $\mathbb{R}^{3}$ :

$$
\langle\phi \mid \psi\rangle=\int d^{3} x \frac{1}{\left(\mathbf{x}^{2}+\lambda^{2}\right)^{3}} \bar{\phi}(\mathbf{x}) \psi(\mathbf{x}) .
$$

Мера в этом скалярном произведении делает нетривиальным определение операторов, необходимых для задачи Кеплера. Дело в том, что помимо определения этих операторов как эрмитовых, нам необходимо сохранить их алгебраические свойства. В качестве примера рассмотрим определение оператора К. Если мы определим квантовый оператор следующим образом:

$$
K_{i}=\frac{1}{2 \lambda}\left[\left(\mathbf{x}^{2}-\lambda^{2}\right) p_{i}-2 x_{i} \mathbf{x p}\right]
$$

где оператор $\mathbf{p}=-i \partial / \partial \mathbf{x}$ (заметим, что $\mathbf{p}$ неэрмитов), то оператор $\mathbf{K}$ будет эрмитовым и его коммутационные соотношения окажутся такими же, как и в классическом случае:

$$
\left[K_{i}, K_{j}\right]=i \epsilon_{i j k} L_{k}
$$

Определение оператора $\mathbf{L}$ не представляет трудностей, поскольку он коммутирует с мерой в (10). Имея операторы $\mathbf{K}, \mathbf{L}$, мы можем правильно определить кинетическую часть квантового гамильтониана

$$
\mathbf{K}^{2}+\mathbf{L}^{2}=-\frac{\left(\mathbf{x}^{2}+\lambda^{2}\right)^{3}}{4 \lambda^{2}} \frac{\partial}{\partial x_{i}} \frac{1}{\mathbf{x}^{2}+\lambda^{2}} \frac{\partial}{\partial x_{i}},
$$

который, очевидно, оказывается эрмитовым относительно (10). Таким образом, полный гамильтониан имеет следующий вид:

$$
H=\frac{2 \lambda^{2}}{m}\left[\mathbf{K}^{2}+\mathbf{L}^{2}+\frac{\alpha}{\lambda} \frac{\mathbf{x}^{2}-\lambda^{2}}{|\mathbf{x}|}\right]=\frac{2 \lambda^{2}}{m} h .
$$


Теперь мы можем определить квантовый вектор Лапласа-Рунге-Ленца

$$
\mathbf{A}=\frac{1}{2}(\mathbf{K} \times \mathbf{L}-\mathbf{L} \times \mathbf{K})+\alpha \frac{\mathbf{x}}{|\mathbf{x}|} .
$$

Определенный таким образом оператор $\mathbf{A}$ коммутирует с гамильтонианом: $[h, \mathbf{A}]=0$. Коммутатор компонент $\mathbf{A}$ оказывается таким же, как и в классическом случае:

$$
\left[A_{i}, A_{j}\right]=-i \epsilon_{i j k} L_{k}\left(h-2 \mathbf{L}^{2}\right),
$$

a $\mathbf{A}^{2}$ несколько отличается от классического аналога:

$$
\mathbf{A}^{2}=\alpha^{2}+h\left(\mathbf{L}^{2}+1\right)-\left(\mathbf{L}^{2}+1\right)^{2}+1 .
$$

Теперь мы займемся поиском оператора $\mathbf{R}$ такого, что

$$
\left[R_{i}, R_{j}\right]=i \epsilon_{i j k} L_{k}
$$

в форме, не разрушающей эрмитовости:

$$
R_{i}=f^{1 / 2}\left(\mathbf{L}^{2}\right) A_{i} f^{1 / 2}\left(\mathbf{L}^{2}\right) .
$$

Далее нам будет удобно использовать вместо оператора $\mathbf{L}^{2}$ его функцию

$$
\gamma=\sqrt{\left(\mathbf{L}^{2}+\frac{1}{4}\right)}-\frac{1}{2}, \quad \mathbf{L}^{2}=\gamma(\gamma+1) .
$$

Также нам понадобится следующая формула, доказанная в приложении, справедливая для любого векторного оператора $\mathbf{A}$ такого, что $\mathbf{A L}=0$ :

$$
A_{i} f(\gamma)=\frac{f(\gamma+1)}{2 \gamma+1}\left[(\gamma+1) A_{i}+i \epsilon_{i j k} L_{j} A_{k}\right]+\frac{f(\gamma-1)}{2 \gamma+1}\left[\gamma A_{i}-i \epsilon_{i j k} L_{j} A_{k}\right]
$$

Теперь можно вычислить коммутатор (11):

$$
\left[f^{1 / 2}(\gamma) A_{i} f^{1 / 2}(\gamma), f^{1 / 2}(\gamma) A_{j} f^{1 / 2}(\gamma)\right]=f^{1 / 2}(\gamma)\left(A_{i} f(\gamma) A_{j}-A_{j} f(\gamma) A_{i}\right) f^{1 / 2}(\gamma)
$$

Для вычисления выражения в скобках в правой части равенства (13) мы используем уравнение (12) и после алгебраических преобразований получаем соотношение

$$
\left(A_{i} f(\gamma) A_{j}-A_{j} f(\gamma) A_{i}\right)=i \epsilon_{i j k} L_{k}\left(\frac{f(\gamma+1)}{2 \gamma+1}\left(\gamma r(\gamma)-\mathbf{A}^{2}\right)+\frac{f(\gamma-1)}{2 \gamma+1}\left((\gamma+1) r(\gamma)+\mathbf{A}^{2}\right)\right)
$$

где мы ввели функцию $r(\gamma)$ с учетом равенства

$$
\left[A_{i}, A_{j}\right]=i \epsilon_{i j k} L_{k} r(\gamma)
$$

Используя этот результат, можно продолжить вычисления коммутатора (11) и переместить все функции $f^{1 / 2}(\gamma)$ направо, поскольку они коммутируют с $\mathbf{L}$ :

$$
\left[R_{i}, R_{j}\right]=i \epsilon_{i j k} L_{k} T
$$

5 Теоретическая и математическая физика, т. 155, № 2, 2008 г. 
где $T$ задается равенством

$$
T=\frac{f(\gamma) f(\gamma+1)}{2 \gamma+1}\left(\gamma r(\gamma)-\mathbf{A}^{2}\right)+\frac{f(\gamma) f(\gamma-1)}{2 \gamma+1}\left((\gamma+1) r(\gamma)+\mathbf{A}^{2}\right) .
$$

Наша цель - добиться выполнения равенства $T=1$. Имея выражение для $r(\gamma)$ и $\mathbf{A}^{2}$ :

$$
\begin{gathered}
r(\gamma)=2 \gamma(\gamma+1)-h, \\
\mathbf{A}^{2}=\alpha^{2}+h(\gamma(\gamma+1)+1)-\gamma^{2}(\gamma+1)^{2}-2 \gamma(\gamma+1),
\end{gathered}
$$

мы можем переписать соотношение $T=1$ в следующей форме:

$$
\begin{aligned}
& f(\gamma) f(\gamma+1)\left[\alpha^{2}+h(\gamma+1)^{2}-(\gamma+1)^{2}\left((\gamma+1)^{2}-1\right)\right]- \\
& -f(\gamma) f(\gamma-1)\left[\alpha^{2}+h \gamma^{2}-\gamma^{2}\left(\gamma^{2}-1\right)\right]=-(2 \gamma+1) .
\end{aligned}
$$

Первый и второй члены в левой части равенства (14) отличаются сдвигом $\gamma$ на единицу, поэтому мы немедленно приходим к уравнению

$$
f(\gamma) f(\gamma-1)=\frac{\mu-\gamma^{2}}{\alpha^{2}+h \gamma^{2}-\gamma^{2}\left(\gamma^{2}-1\right)},
$$

где $\mu$ не зависит от $\gamma$. Прежде чем решать уравнение $(15)$ для $f(\gamma)$, вычислим квадрат вектора $\mathbf{R}$,

$$
\mathbf{R}^{2}=f^{1 / 2}(\gamma) A_{i} f(\gamma) A_{i} f^{1 / 2}(\gamma)
$$

Используя опять уравнение (12), мы после алгебраических преобразований получаem

$$
\mathbf{R}^{2}=\frac{f(\gamma) f(\gamma+1)}{2 \gamma+1}(\gamma+1)\left[\mathbf{A}^{2}-\gamma r(\gamma)\right]+\frac{f(\gamma) f(\gamma-1)}{2 \gamma+1} \gamma\left[\mathbf{A}^{2}+(\gamma+1) r(\gamma)\right]
$$

Теперь, учитывая результат (15), перепишем уравнение в следующем виде:

$$
\mathbf{R}^{2}=\mu-1-\gamma(\gamma+1)
$$

Следовательно, имеем

$$
\mathbf{R}^{2}+\mathbf{L}^{2}=\mu-1
$$

т.е. тот результат, который мы и ожидали. Теперь выберем $\mu$. Уравнение (15) может быть записано в виде

$$
f(\gamma) f(\gamma-1)=\frac{\mu-\gamma^{2}}{\left(k_{1}-\gamma^{2}\right)\left(\gamma^{2}-k_{2}\right)}, \quad k_{1,2}=\frac{h+1}{2} \pm \sqrt{\frac{(h+1)^{2}}{4}+\alpha^{2}} .
$$

Эта формула напоминает нам классический случай. Положим

$$
\mu=\frac{h+1}{2}+\sqrt{\frac{(h+1)^{2}}{4}+\alpha^{2}} .
$$

Тогда формулу (16) запишем в следующем виде:

$$
\mathbf{R}^{2}+\mathbf{L}^{2}=-1+\frac{h+1}{2}+\sqrt{\frac{(h+1)^{2}}{4}+\alpha^{2}}
$$


и, как следствие, получим выражение для гамильтониана через $\mathbf{R}^{2}+\mathbf{L}^{2}$ :

$$
h=\mathbf{R}^{2}+\mathbf{L}^{2}-\frac{\alpha^{2}}{\mathbf{R}^{2}+\mathbf{L}^{2}+1} .
$$

Обсудим полученное представление для гамильтониана. Операторы $\mathbf{R}$ и $\mathbf{L}$ образуют алгебру $S O(4)$, которая является прямой суммой двух алгебр $S O(3)$. Поэтому мы можем ввести вместо $\mathbf{R}$ и $\mathbf{L}$ другую пару операторов

$$
\mathbf{M}=\frac{1}{2}(\mathbf{L}+\mathbf{R}), \quad \mathbf{N}=\frac{1}{2}(\mathbf{L}-\mathbf{R})
$$

таких, что

$$
\left[M_{i}, M_{j}\right]=i \epsilon_{i j k} M_{k}, \quad\left[N_{i}, N_{j}\right]=i \epsilon_{i j k} N_{k}, \quad\left[M_{i}, N_{j}\right]=0 .
$$

Два оператора Казимира алгебры $S O(4)$ в терминах $\mathbf{M}, \mathbf{N}$ имеют вид $C_{1}=\mathbf{M}^{2}$, $C_{2}=\mathbf{N}^{2}$. Поскольку $\mathbf{R L}=0$, имеем

$$
C_{1}=C_{2}=\frac{1}{4}\left(\mathbf{R}^{2}+\mathbf{L}^{2}\right)
$$

Спектр $C_{1}\left(C_{2}\right)$ дается известной формулой $k(k+1), k=0,1 / 2,1, \ldots$ Представления, характеризуемые $k$, содержат все угловые моменты $l=0,1, \ldots, 2 k$ с кратностью, равной единице. В результате спектр гамильтониана $h$ имеет вид

$$
h=4 k(k+1)-\frac{\alpha^{2}}{4 k(k+1)+1}=2 k(2 k+2)-\frac{\alpha^{2}}{(2 k+1)^{2}} .
$$

Вводя другое квантовое число $n=2 k+1, n=1,2, \ldots$, мы можем представить (18) в форме

$$
h=(n-1)(n+1)-\frac{\alpha^{2}}{n^{2}},
$$

что совпадает с результатом Шредингера [1].

Последний вопрос, который должен быть решен, - это получение явной формулы для функции $f(\gamma)$. В принципе мы можем обойтись и без этой формулы. Используя уравнение (12), можно построить вектор $\mathbf{R}$, зная только билинейные комбинации $f(\gamma) f(\gamma \pm 1)$. Однако для полноты картины мы приведем и эту формулу.

$\mathrm{C}$ нашим выбором $\mu$ (см. (17)), уравнение (15) принимает следующий вид:

$$
f(\gamma) f(\gamma-1)=\frac{1}{\gamma^{2}+\sqrt{\frac{(h+1)^{2}}{4}+\alpha^{2}}-\frac{h+1}{2}} .
$$

Выражение

$$
\sqrt{\frac{(h+1)^{2}}{4}+\alpha^{2}}-\frac{h+1}{2}
$$

всегда положительно. Обозначая его $\rho^{2}$, мы имеем

$$
f(\gamma) f(\gamma-1)=\frac{1}{\gamma^{2}+\rho^{2}}
$$


Решение этого уравнения задается формулой

$$
f(x)=\frac{i}{x-i \rho} \frac{\Gamma\left(\frac{x-i \rho+1}{2}\right)}{\Gamma\left(\frac{x-i \rho}{2}\right)} \frac{\Gamma\left(-\frac{x+i \rho}{2}\right)}{\Gamma\left(-\frac{x+i \rho-1}{2}\right)} .
$$

Простейшее доказательство этого результата состоит в прямой подстановке в уравнение (20).

\section{3. ОБСУЖДЕНИЕ}

Итак, мы рассмотрели алгебраический вывод формулы для спектра атома водорода в пространстве постоянной положительной кривизны. Этот спектр замечательным образом представляется в виде суммы спектров свободного движения на $S^{3}$ и дискретной части кулоновского спектра в плоском пространстве. Этот факт был отмечен еще Шредингером. В отличие от плоского случая, спектр этой задачи чисто дискретный. Это обстоятельство позволяет надеяться на возможность построения алгебры, генерирующей спектр. Предварительные рассмотрения показывают, что это некомпактная алгебра $S O(4,2)$. Элементами этой алгебры являются генераторы только что построенной алгебры $S O(4)$, гамильтониан, а также повышающие и понижающие операторы, с помощью которых можно построить все волновые функции, зная волновую функцию основного состояния. Последняя находится очень просто с помощью следующего построения. Из формулы (19) при $n=1$ получаем энергию основного состояния $E_{0}=-\alpha^{2}$. Далее нетрудно показать, что справедливо следующее соотношение:

$$
\left[i\left(K_{j}+L_{j}\right)+\alpha \frac{x_{j}}{|\mathbf{x}|}\right]\left[-i\left(K_{j}+L_{j}\right)+\alpha \frac{x_{j}}{|\mathbf{x}|}\right]=h+\alpha^{2} .
$$

Правая часть этого равенства обращается в нуль при действии на основное состояние. Отсюда мы получаем для основного состояния уравнение первого порядка

$$
\left[-i\left(K_{j}+L_{j}\right)+\alpha \frac{x_{j}}{|\mathbf{x}|}\right] \psi_{0}\left(x_{i}\right)=0,
$$

решением которого является $\psi_{0}\left(x_{i}\right)=e^{-2 \alpha \operatorname{arctg}(|\mathbf{x}| / \lambda)}$.

\section{ПРИЛОЖЕНИЕ}

Рассмотрим функцию $F\left(\mathbf{L}^{2}\right)$, умноженную слева на оператор $\mathbf{A}$, который является вектором по отношению к $\mathbf{L}$ и удовлетворяет условию $\mathbf{A L}=0$. В общем случае справедливо следующее соотношение:

$$
A_{i} F\left(\mathbf{L}^{2}\right)=S\left(\mathbf{L}^{2}\right) A_{i}+T\left(\mathbf{L}^{2}\right) i \epsilon_{i j k} L_{j} A_{k}
$$

где функции $S, T$ определяются функцией $F$. Ограничение $\mathbf{A L}=0$ не является существенным, и нам достаточно рассмотреть только такие операторы. Пусть функция $F\left(\mathbf{L}^{2}\right)$ представлена в виде

$$
F\left(\mathbf{L}^{2}\right)=\int d \alpha \phi(\alpha) e^{i x \alpha \mathbf{L}^{2}}
$$


Тогда для вывода соотношения (П.1) достаточно рассмотреть только случай экспоненциальной зависимости $F\left(\mathbf{L}^{2}\right)$ от $\mathbf{L}^{2}$. Умножая экспоненту слева на $\mathbf{A}$, имеем

$$
A_{i} e^{i \alpha \mathbf{L}^{2}}=f\left(\alpha, \mathbf{L}^{2}\right) A_{i}+g\left(\alpha, \mathbf{L}^{2}\right) i \epsilon_{i j k} L_{j} A_{k},
$$

где функции $f, g$ должны быть определены. Продифференцируем обе части (П.2) по $\alpha$ :

$$
\begin{aligned}
\partial_{\alpha} A_{i} e^{i \alpha \mathbf{L}^{2}}= & i A_{i} e^{i \alpha \mathbf{L}^{2}} \mathbf{L}^{2}=i\left[f\left(\alpha, \mathbf{L}^{2}\right) A_{i}+g\left(\alpha, \mathbf{L}^{2}\right) i \epsilon_{i j k} L_{j} A_{k}\right] \mathbf{L}^{2}= \\
= & i\left[f\left(\alpha, \mathbf{L}^{2}\right)\left(\mathbf{L}^{2}+2\right)+2 g\left(\alpha, \mathbf{L}^{2}\right) \mathbf{L}^{2}\right] A_{i}+ \\
& \quad+i\left[2 f\left(\alpha, \mathbf{L}^{2}\right)+g\left(\alpha, \mathbf{L}^{2}\right)\right] i \epsilon_{i j k} L_{j} A_{k}= \\
= & \partial_{\alpha} f\left(\alpha, \mathbf{L}^{2}\right) A_{i}+\partial_{\alpha} g\left(\alpha, \mathbf{L}^{2}\right) i \epsilon_{i j k} L_{j} A_{k},
\end{aligned}
$$

где мы использовали следующее соотношение:

$$
A_{i} \mathbf{L}^{2}=\left(\mathbf{L}^{2}+2\right) A_{i}+2 i \epsilon_{i j k} L_{j} A_{k} .
$$

Из (П.3) вытекают следующие два уравнения для функций $f, g$ :

$$
\begin{gathered}
\partial_{\alpha} f\left(\alpha, \mathbf{L}^{2}\right)=i\left[f\left(\alpha, \mathbf{L}^{2}\right)\left(\mathbf{L}^{2}+2\right)+2 g\left(\alpha, \mathbf{L}^{2}\right) \mathbf{L}^{2}\right] \\
\partial_{\alpha} g\left(\alpha, \mathbf{L}^{2}\right)=i\left[2 f\left(\alpha, \mathbf{L}^{2}\right)+g\left(\alpha, \mathbf{L}^{2}\right)\right] \\
f\left(0, \mathbf{L}^{2}\right)=1, \quad g\left(0, \mathbf{L}^{2}\right)=0 .
\end{gathered}
$$

Далее удобно ввести вместо $\mathbf{L}^{2}$ оператор

$$
\gamma=\sqrt{\left(\mathbf{L}^{2}+\frac{1}{4}\right)}-\frac{1}{2}, \quad \mathbf{L}^{2}=\gamma(\gamma+1) .
$$

В терминах $\gamma$ решение (П.4) имеет следующий вид:

$$
\begin{aligned}
& f(\alpha, \gamma(\gamma+1))=\frac{1}{2 \gamma+1}\left[(\gamma+1) e^{i \alpha(\gamma+1)(\gamma+2)}+\gamma e^{i \alpha \gamma(\gamma-1)}\right], \\
& g(\alpha, \gamma(\gamma+1))=\frac{1}{2 \gamma+1}\left[e^{i \alpha(\gamma+1)(\gamma+2)}-e^{i \alpha \gamma(\gamma-1)}\right] .
\end{aligned}
$$

Подставляя (П.5) в (П.2), получаем

$$
\begin{gathered}
A_{i} e^{i \alpha \gamma(\gamma+1)}=e^{i \alpha(\gamma+1)(\gamma+2)} \frac{1}{2 \gamma+1}\left[(\gamma+1) A_{i}+i \epsilon_{i j k} L_{j} A_{k}\right]+ \\
+e^{i \alpha \gamma(\gamma-1)} \frac{1}{2 \gamma+1}\left[\gamma A_{i}-i \epsilon_{i j k} L_{j} A_{k}\right] .
\end{gathered}
$$

Таким образом, “пронося" векторный оператор через экспоненту от $\gamma(\gamma+1)$, мы получаем два члена, в одном из которых $\gamma$ сдвигается на +1 , в другом - на -1 . Если бы мы имели оператор $\mathbf{A}$ такой, что $\mathbf{A L} \neq 0$, то появился бы третий член, в котором $\gamma$ не сдвигалась бы совсем. Производя преобразование Фурье, мы распространяем результат на произвольные функции:

$$
\begin{gathered}
A_{i} F(\gamma)=F(\gamma+1) \frac{1}{2 \gamma+1}\left[(\gamma+1) A_{i}+i \epsilon_{i j k} L_{j} A_{k}\right]+ \\
+F(\gamma-1) \frac{1}{2 \gamma+1}\left[\gamma A_{i}-i \epsilon_{i j k} L_{j} A_{k}\right]
\end{gathered}
$$

Аналогичная формула существует также и для умножения справа на оператор $\mathbf{A}$. 
Благодарности. Автор выражает свою признательность А.К. Лиходеду и А. В. Разумову за их замечания и плодотворные дискуссии, М. Сантандеру за то, что он привлек внимание автора к этой проблеме. Работа была поддержана программой ENTER-2004/04EP-48 Европейского фонда и Министерства развития Греции и РФФИ (грант № 07-01-00234).

\section{Список литературы}

[1] E. Schrödinger, Proc. Roy Irish Acad. Sect. A, 46 (1940), 9-16.

[2] L. M. Nieto, H. C. Rosu, M. Santander, Modern Phys. Lett. A, 14:35 (1999), 2463-2469; arXiv: quant-ph/9911010.

[3] V. Fock, Z. Phys., 98:3-4 (1935), 145-154.

[4] W. Pauli Jr., Z. Phys., 36:5 (1926), 336-363.

Поступила в редакцию 21.10.2007 